\title{
MODELOS DE REGRESSÃO ALEATÓRIA ATRAVÉS DO PESO CORPORAL EM CURVAS DE CRESCIMENTO DE AVES
}

\author{
Milena Vieira Lima²; Jeferson Corrêa Ribeiro'; Lorrayne Gomes²; Andreia Santos Cezário ${ }^{1}$, Eliandra \\ Maria Bianchini Oliveira ${ }^{1}$; Wallacy Barbacena Rosa dos Santos ${ }^{1}$; Tiago Neves Pereira Valente ${ }^{3}$; Aline \\ Sousa Camargos ${ }^{1}$
}

${ }^{1}$ Instituto Federal Goiano - IFG, Departamento de Zootecnia, Campus Morrinhos, Morrinhos, GO. ${ }^{2}$ Instituto Federal Goiano - IFG, curso de Agronomia, Campus Morrinhos, Morrinhos, GO. ${ }^{3}$ Instituto Federal Goiano - IFG, Campus Posse. E-mail: jeferson.ribeiro@ifgoiano.edu.br

\section{RESUMO}

Nas avaliações genéticas modernas, os modelos de regressão aleatória têm sidos usados como uma frequente ferramenta, para análise de características longitudinais, como aquelas envolvidas no crescimento animal. Tais características, como o peso corporal, são de fácil mensuração, e possuem excelente resposta à seleção, sendo uma característica importante nos programas de melhoramento animal. Objetivou-se com essa revisão discutir os diferentes modelos de regressão aleatória, que estão envolvidos no crescimento de aves. Os modelos de regressão aleatória foram propostos como uma alternativa para avaliação genética de características que são medidas repetidamente na vida do animal. Esses modelos permitem a predição de coeficientes de regressão que representam o comportamento do valor genético aditivo de cada animal na característica avaliada em função do tempo (idade). Desta forma, infinitos valores da variável independente são considerados dentro de um intervalo definido, através dos desvios de cada animal em relação a uma curva fixa estimada. As estimativas dos componentes de covariância atribuídos aos coeficientes de regressão aleatórios permitem estimar a covariância entre quaisquer valores da variável independente para o efeito aleatório modelado, o que é realizado por meio da função de covariância. Assim, os modelos de regressão aleatória otimizam a utilização de informações de pesagens, considerando a estrutura de covariâncias entre as idades abrangidas na avaliação e possibilitam a descrição componentes de variância estimados envolvidos no crescimento, além de possibilitar pressuposições para outros da curva, dentro do intervalo de estimação.

Palavras-chave: avaliação genética; dados longitudinais; medidas repetidas; polinômio de Legendre; spline

\section{RANDOM REGRESSION MODELS THROUGH BODY WEIGHT IN GROWTH CURVES OF POULTRY}

\begin{abstract}
In modern genetic evaluations, random regression models have been used as a custom tool in order to analyze longitudinal traits such as the ones involved in animal growth. Such traits as body weight have an easy mensuration and an excellent response to selection, which is a suitable and important feature for animal breeding programs. The purpose of this review is to discuss about different random regression models that are involved in farm bird growth. The random regression models are recommended as an alternative to genetic evaluation of traits that are regularly measured during the animal life. These models allow the prediction of regression coefficients that represent the behavior of the additive genetic value for each animal in the specific evaluated trait in relation to time (age). Thus, interminable values for the independent variable are considered
\end{abstract}


within a defined interval, through deviations of each animal in relation to an estimated and fixed curve. The covariance component estimates assigned to random regression coefficients allow the covariance estimation between any values of the independent variable for a modeled random effect, which is accomplished by the covariance function. Therefore, random regression models improve the use of the weight information, when covariance structures between the studied ages are taken into account during the evaluation. They also allow the description of the estimated variance components that are involved in growth, besides granting presumptions for others in the curve inside the interval estimation.

Keywords: genetic evaluation; Legendre polynomial; longitudinal data; regular measures; spline

\section{INTRODUÇÃO}

Nos programas de melhoramento animal, características que influenciam o crescimento animal é de fundamental importância. A busca de animais mais eficientes, e de rápido crescimento corporal e desejável do ponto de vista econômico. Características como peso corporal, tamanho ou dias para atingir determinado peso têm sido largamente utilizadas como forma de determinação da curva de crescimento dos animais.

Várias vezes ao longo da trajetória de crescimento, análises de peso podem ser realizadas de várias maneiras. Uma dessas maneiras, é considerando que o peso varia em diferentes idades e que são medidas repetidas de uma determinada característica, tal como em modelos de repetibilidade. A utilização de modelos de repetibilidade supõem que em diferentes idades, ocorra correlação genética é de ambiente, permanecendo iguais à unidade, significando que cada indivíduo estudado apresente efeito genético aditivo e de ambiente permanente que se repetem em dados coletados em diferentes idades. Entretanto, é possível que alguns animais sejam geneticamente superiores para algumas características, como peso em uma idade, mas não em outra (Oliveira, 2011).

Dentre as características usadas na seleção de animais de produção, o peso se destaca nos programas de melhoramento genético animal para avaliação do crescimento. Por ser de fácil mensuração e excelente resposta à seleção, o peso está presente em todos os programas de melhoramento animal, e, portanto, sendo alvo de estudo em diversas áreas do conhecimento, com o intuito de melhor conhecer o fenômeno por traz dessa variável.

O uso intenso dessa característica ao longo do tempo foi importante para o desenvolvimento das linhagens de alto desempenho em várias espécies animais. Em aves, por exemplo, a seleção tem sido tradicionalmente feita sobre um peso a uma idade fixa, fazendo com que a idade ao abate seja reduzida, no caso de frangos de corte, em um dia a cada geração (Mignon-Grasteau et al., 2001).

Uma das formas de se estudar as funções de crescimento é através de modelos de regressão aleatória (MRA). Os MRAs permitem a predição de coeficientes de regressão que representam o comportamento do valor genético aditivo de cada animal na característica avaliada em função do tempo (idade) ou qualquer outra variável contínua. A utilização de modelos de regressão aleatória tem sido uma alternativa para modelar características de crescimento, que por sua vez, são dados repetidos na vida dos animais, consideradas dados longitudinais. Tais modelos de regressão aleatória permitem agourar determinados valores genéticos para a curva de crescimento com um todo, permitindo que obtenha-se uma melhor utilização de dados, uma vez que todas as medidas do animais e de parentes são utilizados para avaliação (Bonafé et al., 2011). O conhecimento da curva de crescimento permite a estimação dos parâmetros genéticos e ambientais, tanto nas idades mensuradas, quanto nos intervalos da curva, além de permitir a determinação de coeficientes de regressão para esses efeitos (Meyer, 2000). 
Objetivou-se com essa revisão abordar os estudos da curva de crescimento através de modelos de regressão aleatória em aves.

\section{MODELOS DE REGRESSÃO ALEATÓRIA EM ESTUDOS DE CURVA DE CRESCIMENTO}

Os modelos de regressão aleatória (Henderson JR., 1982) têm sidos propostos como alternativa para avaliação genética de características que são medidas repetidamente na vida do animal ou são consideradas como função contínuas, tais como as medidas de peso dos animais. Os modelos de repetibilidade fornecem predições únicas, válidas para todas as idades e ambientes considerados. Os modelos multicaracterísticos resultam em predições para número limitado de idades ou ambientes. Os MRAs permitem a predição de coeficientes de regressão que representam o comportamento do valor genético aditivo de cada animal na característica avaliada em função do tempo (idade) ou qualquer outra variável contínua. Desta forma, infinitos valores da variável independente são considerados dentro de um intervalo definido. Por este motivo, o modelo não exige utilização de fatores de ajustes para idades consideradas como padrão ou projeções de lactações parciais para produção total e permite uma melhor utilização dos dados disponíveis, já que todas as informações do animal e de seus parentes são utilizadas. Os MRAs baseiam-se na estimação de uma curva fixa para o conjunto de dados e a curva de regressão de cada animal represente um desvio da curva fixa. As estimativas dos componentes de covariância atribuídos aos coeficientes de regressão aleatórios permitem estimar a covariância entre quaisquer valores da variável independente para o efeito aleatório modelado, o que é realizado por meio da função de covariância. Esta função descreve toda a estrutura de covariância deste efeito para o intervalo considerado na variável independente (Silva et al., 2008).

Trabalhando com dados simulados de crescimento de bovinos de corte, Meyer (2004) observou que os modelos de regressão aleatória apresentaram acurácias, medidas pela correlação entre valores simulados com estimados, de 5,7 a 8,3\% maiores que as obtidas com os modelos multicaracterísticos. A autora afirma que uma vantagem adquirida pela abordagem da regressão aleatória está na modelagem das variâncias e outros parâmetros genéticos, obtidos através desse modelo. Na realidade, os modelos de regressão aleatória proporciona melhor aproveitamento das informações que são atualmente coletadas, as quais têm sido utilizadas apenas para ajustar os pesos para idades consideradas como "padrão". Se considerarmos que registros de pesos dos animais, embora seja de fácil obtenção, tem elevado custo operacional e monetário, a utilização deste modelo pode resultar em importante retorno econômico, pois com o mesmo investimento, os animais seriam avaliados com maior acurácia.

\section{ASPECTOS TEÓRICOS DOS MODELOS DE REGRESSÃO ALEATÓRIA}

Segundo Silva et al. (2008), os modelos de regressão aleatória são modelados através dos desvios de cada animal em relação a uma curva fixa estimada. As estimativas dos componentes de covariância atribuídos aos coeficientes de regressão aleatórios permitem estimar a covariância entre quaisquer valores da variável independente para o efeito aleatório modelado, o que é realizado por meio da função de covariância. Esta função descreve toda a estrutura de covariância deste efeito para o intervalo considerado na variável independente.

Podemos considerar então que a relação entre crescimento e idade ( $/$ ) é curvilínea e o seguinte modelo pode ser ajustado, onde a observação $k$ no animal $j$ pode ser descrita como:

$y_{j k}=\alpha_{0 j}+\alpha_{1 j}(I)+\alpha_{2 j}(I)^{2}+\varepsilon_{j k}(1)$

que pode ser expandida considerando um modelo para cada $\alpha_{i j}$, como segue:

$$
\alpha_{i j}=\mu_{i}+\alpha_{i j}+p_{i j}+e_{i j}(2)
$$


em que: $\mu_{i}$ é a média do efeito;

$\alpha_{i j}$ é o efeito genético aditivo aleatório;

$p_{i j}$ é o efeito ambiental permanente aleatório;

$e_{i j}$ é o efeito residual aleatório

Este submodelo simplificado ilustra as principais vantagens dos modelos de regressão aleatória. Cada $\alpha_{i j}$ na equação de regressão que relaciona crescimento e idade pode ser substituído pelo submodelo descrito, o que permite obter:

$$
\begin{aligned}
y_{j k} & =\left(\mu_{0}+a_{o j}+p_{0 j}+e_{0 j}\right) \\
& +\left(\mu_{1}+a_{1 j}+p_{1 j}+e_{1 j}\right)(I) \\
& +\left(\mu_{2}+a_{2 j}+p_{2 j}+e_{2 j}\right)(I)^{2} \\
& +\varepsilon_{j k}
\end{aligned}
$$

Os termos neste modelo podem ser rearranjados para fornecer a seguinte equação:

$$
\begin{aligned}
y_{j k} & =\left[\mu_{0}+\mu_{1}(I)+\mu_{2}(I)^{2}\right] \\
& +\left(a_{0 j}+a_{1 j}(I)+a_{2 j}(I)^{2}\right) \\
& +\left[p_{0 j}+p_{1 j}(I)+p_{2 j}(I)^{2}\right] \\
& +\left[e_{0 j}+e_{1 j}(I)+e_{2 j}(I)^{2}\right] \\
& +\varepsilon_{j k}
\end{aligned}
$$

Esta equação é composta dos seguintes termos:

$$
y_{j k}=\text { (regressão fixa) }
$$

+(regressão aleatória para efeito genético)

+(regressão aleatória para efeito ambiente permanente) (5)

+ (regressão aleatória para efeito residual)

+ (termo do erro)

Quando modelos de regressão aleatória são desenvolvidos desta forma, as funções de regressão de efeitos aleatórios utilizam os mesmos polinômios da função de regressão que ajusta os dados fenotípicos (constante, idade e idade ao quadrado). Isto sugere que as funções de regressão fixa e aleatória deveriam ser da mesma forma em um modelo de regressão aleatória, o que não é obrigatório.

O modelo na forma matricial para dados de crescimento é representado por:

$$
y=X \beta+Z_{1} a+Z_{1} p+Z_{2} e+\varepsilon
$$

em que: $y$ é o vetor de observações dos pesos em diferentes idades para os diferentes indivíduos analisados; $X$ é a matriz de incidência dos coeficientes de regressão fixos; $Z_{1}$ matriz de covariáveis relativas ao efeito genético aditivo e ambiental permanente; $Z_{2}$ é a matriz de covariáveis relativas ao efeito residual; $\beta$ é o vetor que contém os efeitos fixos do modelo; $a$ é o vetor de coeficientes de regressão aleatória atribuídos aos efeitos genéticos aditivos; $\rho$ vetor dos coeficientes de regressão aleatórios atribuídos aos efeitos ambientais permanentes do animal; $e$ vetor dos coeficiente de regressão aleatórios atribuídos aos efeitos residuais; $\varepsilon$ é o vetor de resíduos associados a $y$. 
Assumindo $\left[\begin{array}{l}a \\ c \\ e\end{array}\right] \sim N(0, V), V=\left[\begin{array}{lll}K_{a} \otimes A & 0 & 0 \\ 0 & K_{c} \otimes I & 0 \\ 0 & 0 & R\end{array}\right]$

em que: $K_{a}$ é a matriz de (co)variâncias entre os coeficientes de regressão aleatória aditivo; $A$ é a matriz de numeradores do coeficientes de parentesco entre os animais; $K_{c}$ é a matriz de (co)variâncias entre os coeficientes de regressão aleatória de ambiente permanente; I é uma matriz identidade; $R$ é a matriz diagonal de variâncias residuais com elementos que dependem do número de classes ajustadas.

A partir da equação 5, podemos observar que para cada animal, uma curva será traçada de acordo com os todos os termos contidos no modelo, ou seja, será definida uma curva para o efeito aditivo genético, uma curva para o efeito de ambiente permanente, uma curva pra o efeito residual, etc. Cada curva será composta pelo intercepto, $I$ e $I^{2}$. Para o ajuste dessas curvas, são usados polinômios de Legendre ou polinômios segmentados (Splines).

As funções ortogonais polinomiais de Legendre ou, como é mais popularmente conhecido, polinômios de Legendre são mais um tipo de funções polinomiais que possuem propriedades interessantes, quando se deseja ajustar um conjunto de variáveis ou de amostras: são ortogonais no intervalo de -1 a 1 . Os polinômios de Legendre são soluções da equação diferencial do matemático francês Adrien-Marie Legendre, sendo:

$$
\frac{d}{d x}\left[\left(1-x^{2}\right) \frac{d}{d x} P_{n}(x)\right]+n(n+1) P_{n}(x)=0
$$

Segundo Schaeffer (2009), os polinômios ortogonais tendem a reduzir as correlações entre os coeficientes de regressão estimados, evitando problemas de multicolinearidade severa, o que pode gerar impedimentos quando se utiliza métodos de REML ou bayesianos, e permitindo uma maior velocidade no processo de convergência. Além disso, esses polinômios possuem maiores facilidade de cálculos e utilização.

A escolha do grau do polinômio pode ser definida por critérios como o teste da razão de verossimilhança, critério de informação de Akaike (AIC) e o critério bayesiano de Schwarz (BIC), entre outros. Em alguns casos, os polinômios de menor ordem podem ser utilizados para o ajuste de funções e essa redução pode ser feita observando os autovalores da matriz $\mathrm{H}$, de menor ordem que matriz $G$ e fazendo o teste $F$ para verificação do ajuste (Kirkipatick et al., 1990). Lembrando que na teoria do melhoramento animal, $G$ é a matriz de variância e covariância genética e $\mathrm{H}$ é a matriz que combina a matriz de relacionamento genético ou de parentesco (matriz $A$ ) com a matriz $G$.

Funções Splines ou polinômios segmentados são pequenos segmentos de polinômios de menor grau unidos em regiões conhecidas por nós (knots). Esses nós conectam os segmentos de forma a permitir a obtenção de derivadas contínuas de grau $n-1$ (Schenkel, 1989). Os valores da função e as primeiras $n-1$ derivadas devem coincidir no(s) nó(s), portanto, a função spline é uma função contínua com $n-1$ derivadas contínuas (Paulson, 2007).

Em um sentido real, Splines é uma evolução da inferência paramétrica clássica e uma ponte entre os métodos paramétricos e não paramétricos. Enquanto as Splines não são paramétricas na forma funcional, na maioria dos casos, podem ser escritos como uma combinação linear de funções de base que geralmente têm uma representação polinomial. Assim, existem uma grande quantidade de classes de funções possíveis de serem utilizadas com a vantagem adicional de utilizar propriedade que permitem obter curvas mais suaves (Wegman; Wright, 1983).

O grande obstáculo dos Splines é o conhecimento dos nós. O conhecimento prévio do 
pesquisador na área técnica específica, o uso de diagramas e médias para a estimação grosseira de nós ou o uso de um modelo não linear para a estimação simultânea dos nós e dos coeficientes de regressão podem ser empregados nesta tarefa. O que tem sido feito na prática é a fixação dos nós por inspeção visual, ou seja, plota-se o conjunto de dados em um gráfico de dispersão, e a partir desse ponto, o pesquisador determina quais são os pontos críticos e locais onde devem inserir os nós. Para assegurar uma escolha mais correta (que minimize a soma de quadrados dos resíduos) podem-se escolher pontos próximos aos locais onde poderia estar situado o nó e então proceder à estimação dos parâmetros do modelo. $O$ ponto adotado seria aquele que minimizasse a soma de quadrados do resíduo. Segundo Schenkel (1989), este método, aparentemente trabalhoso, é exequível e bastante prático em decorrência da facilidade de computação dos polinômios.

Outra vantagem para o uso funções Spline ou polinômios de Legendre é que as matrizes formadas pelos MRAs são mais esparsas, ou seja, são mais fáceis para a inversão. Para uma função Spline de grau $p$, cada linha da matriz terá $p+1$ coeficientes, e as exigências computacionais são menores, do que análises correspondentes com o mesmo número de coeficientes para uma base polinomial.

\section{ALGUNS EXEMPLOS DE CURVA DE CRESCIMENTO USANDO MODELOS DE REGRESSÃO ALEATÓRIA EM AVES}

Trabalhos envolvendo modelos de regressão aleatória para dados de crescimento em aves são escassos na literatura. Tholon e Queiroz (2008), analisou dados de 7.369 registros de peso corporal medidos desde o nascimento até 210 dias de perdizes nascidos entre 2000 e 2004, com o objetivo de modelar a estrutura das variâncias residuais heterogêneas para cada idade, usando polinômios de Legendre para modelar os efeitos aleatórios do modelo. $O$ modelo que se mostrou mais parcimonioso continha um polinômio de Legendre de ordem 6 para efeitos fixos, 6 para efeitos genético aditivo e 6 para efeito de ambiente permanente.

Em um estudo, Bonafé et al. (2011) analisaram dados de 26.835 e 27.447 observações de crescimento, de 3.909 e 4.040 codornas de corte das linhagens UFV1 e UFV2, respectivamente, com o objetivo de avaliar o melhor modelo de regressão aleatória que melhor se ajusta ao estudo genético da curva de crescimento dos animais. O peso corporal nas duas linhagens foi avaliado aos $1,7,14,21,28,35$ e 42 dias de idade. Ordens de ajuste das funções contínuas foram gradualmente aumentadas (ordens variando de 3 a 6) para determinação da ordem mínima necessária para descrever as estruturas de covariância em função do tempo nos modelos de regressão aleatória. A função polinomial de Legendre com as ordens 6 para efeito genético aditivo direto e 5 para efeito permanente de animal, para a linhagem UFV1, e 6 para ambos os efeitos aleatórios, para a linhagem UFV2, foram os mais adequados para descrever a curva de crescimento dos animais.

Rovadoscki et al. (2016) com o objetivo de comparar modelos com diferentes ordens de ajuste por meio de funções polinomiais de Legendre, sob modelos de regressão aleatória, utilizou dados de peso corporal desde a eclosão até os 84 dias de idade $(n=34.730)$ de quatro linhagens experimentais de frangos caipiras. O modelo considerado mais parcimonioso foi o que continha polinômios de Legendre de segunda ordem, tanto para efeitos fixos, quanto para efeitos aleatórios.

Karami, et al. (2017) utilizou 11.826 registros de 2.489 codornas, para estimar parâmetros genéticos para peso corporal de codornas japonesas, usando modelos de regressão aleatória, até aos 49 dias de idade. Os autores utilizaram 19 modelos para identificar as melhores ordens de polinômios de Legendre. Os resultados indicaram um modelo com polinômio de Legendre de ordem 3 para efeitos genéticos aditivos, a ordem 3 para efeitos ambientais permanentes e a ordem 1 para efeitos ambientais permanentes maternos.

Outros trabalhos envolvendo regressão aleatória com dados longitudinais em outras 
espécies, com ajuste dos modelos por meio de polinômios ortogonais de Legendre podem ser consultados em Bohmanova et al., (2008); Bignardi et al., (2008); Chen et al., (2011); Menezes, et al. (2011); Steri et al., (2012); Pereira et al., (2013) Peixoto et al. (2014); Dornelles et al. (2016).

\section{CONCLUSÃO}

Os modelos de regressão aleatória otimizam a utilização de informações de pesagens, considerando a estrutura de covariâncias entre as idades abrangidas na avaliação e possibilitam a descrição dos componentes envolvidos no crescimento, ou seja, permite fazer pressuposições sobre a variável em questão, mesmo que o dia escolhido não tenha sido a data de coleta dos dados.

\section{REFERÊNCIAS}

BIGNARDI, A.B.; EL FARO, L.; ALBUQUERQUE, L.G.; TORRE JÚNIOR, R.A.A. Modelos de regressão aleatória empregando funções B-splines para modelar a produção de leite de vacas da raça Holandesa. In: Reunião Anual da Sociedade Brasileira De Zootecnia, 45, 2008, Lavras. Anais... Lavras: SBZ, 2008. (CD-ROM).

BOHMANOVA, J.; MIGLIOR, F.; JAMROZIK, J; MISZTAL, I; SULLIVAN P.G.; Comparison of random regression models with Legendre polynomials and linear Splines for production traits and somatic cell score of Canadian Holstein cows. Journal of Dairy Science, v.91, p.3627-3638, 2008. https://doi.org/10.3168/jds.2007-0945

BONAFÉ, C.M.; TORRES, R.A.; SARMENTO, J.L.R.; SILVA, L.P.; RIBEIRO, J.C.; TEIXEIRA, R.B.; SILVA, F.G.; SOUSA, M.F.; Modelos de regressão aleatória para descrição da curva de crescimento de codornas de corte. Revista Brasileira de Zootecnia, v.40, n.4, p.765-771, 2011. https://doi.org/10.1590/S1516-35982011000400009

CHEN, C.Y.; MISZTAL, I.; TSURUTA, S.; HERRING, W.O.; HOLL, J.; CULBERTSON M.; Genetic analyses of stillbirth in relation to litter size using random regression models. Journal of Animal Science, v.88, p.3800-3808, 2010. https://doi.org/10.2527/jas.2009-2413

DORNELLES, M.A.; RORATO, P.R.N.; GAMA, L.T.L.; BREDA, F.C.; BONDAN, C.; EVERLING, D.M.; MICHELOTTI, V.T.; FELTES, G.L. Random regression models using different functions to estimate genetic parameters for milk production in Holstein Friesians. Ciência Rural, v.46, n.9, p.1649-1655, 2016. https://doi.org/10.1590/0103-8478cr20150473

HENDERSON Jr.; C.R.; Analysis of covariance in the mixed model: higher-level, nonhomogeneous, and random regressions. Biometrics, v.38, p.623-640, 1982. https://doi.org/10.2307/2530044

KARAMI, K.; ZEREHDARAN, S.; TAHMOORESPUR, M.; BARZANOONI, B.; LOTFI, E. Genetic evaluation of weekly body weight in Japanese quail using random regression models. British Poultry Science, v.58, p.13-18, 2017. https://doi.org/10.1080/00071668.2016.1236362

KIRKPATRICK, M.; LOFSVOLD, D.; BULMER, M.; Analysis of the inheritance, selection and evolution of growth trajectories. Genetics, v.124, p.979-993, 1990.

MEYER, K. Random regression to model phenotypic variation in monthly weights of Australian beef cows. Livestock Production Science, v. 65, p. 19-38, 2000. https://doi.org/10.1016/S0301$\underline{6226(99) 00183-9}$ 
MENEZES, G.R.O.; TORRES, R.A.; SARMENTO, J.L.R.; RODRIGUES, M.T.; BRITO, L.F.; LOPES, P.S.; SILVA, F.G. Modelos de regressão aleatória na avaliação da produção de leite em cabras da raça Saanen. Revista Brasielira de Zootecnia, v.40, n.7, p.1526-1532, 2011 https://doi.org/10.1590/S1516-35982011000700018

MEYER, K.; Scope for a random regression model in genetic evaluation of beef cattle for growth. Livestock Production Science, v.86, p.69-83, 2004. https://doi.org/10.1016/S0301-6226(03)00142$\underline{8}$

MIGNON-GRASTEAU, S.; BEAUMONT, C.; RICARD, F.H. Genetic Analysis of a Selection Experiment on the Growth Curve of Chickens. Poultry Science, v.80 p.849-854, 2001. https://doi.org/10.1093/ps/80.7.849

OLIVEIRA, J.A. Avaliação do crescimento e de modelos de regressão aleatória em caprinos da raça Alpina. 2011. 90 f. Dissertação (Mestrado em Zootecnia) - Universidade Federal de Viçosa, Viçosa.

PAULSON, D.S. Handbook of regression and modeling - Application for the clinical and pharmaceutical industries. New York: Chapman \& Hall / CRC, 2007.

PEIXOTO, M.G.C.D.; SANTOS, D.J.A.; BORQUIS, R.R.A.; BRUNELI, F.A.T.; PANETTO, J.C.C.; TONHATI, $\mathrm{H}$. Random regression models to estimate genetic parameters for milk production of Guzerá cows using orthogonal Legendre polynomials. Pesquisa Agropecuária Brasileira, v.49, n.5, p.372-383, 2014. https://doi.org/10.1590/S0100-204X2014000500007

PEREIRA, R.J.; BIGNARDI, A.B.; EL FARO, L.; VERNEQUE, R.S.; VERCESI FILHO, A.E.; ALBUQUERQUE, L.G.; Random regression models using Legendre polynomials or linear Splines for test-day milk yield of dairy Gyr (Bos indicus) cattle. Journal of Dairy Science, v.96, p.565-574, 2013. https://doi.org/10.3168/ids.2011-5051

ROVAdOSCKI, G.A.; PETRINI, J.; RAMIREZ-DIAZ, J.; PERTILE, S.F.N.; PERTILLE, F.; SALVIAN, M.; LUNG, L.H.S.; RODRIGUEZ, M.A.P.; ZAMPAR, A.; GAYA, L.G.; CARVALHO, R.S.B.; COELHO, A.A.D.; SAVINO, V.J.M.; COUTINHO, L.L.; MOURÃO, G.B. Genetic parameters for growth characteristics of free-range chickens under univariate random regression models. Poultry Science, v.95, n.9, p.1989-1998, 2016. https://doi.org/10.3382/ps/pew167

SCHAEFFER L. R., 2016. Random models. < http://www.aps.uoguelph.ca/ /rs/BOOKS/rrmbook.pdf>. Acessado em fevereiro de 2016.

SCHENKEL F.S. Utilização de polinômios segmentados na pesquisa zootécnica: considerações teóricas e práticas [notas]. Porto Alegre (RS):UFRGS; 1989

SILVA, M.A.; Modelos Lineares Aplicados ao Melhoramento Genético Animal. In: THIÉBAUT, J.T.L; VALENTE, B.D.; TORRES, R.A.; FARIA, F.J.C.; Belo Horizonte: FEPMVZ, 2008, p.322-323

STERI, R.; DIMAURO, C.; CANAVESI, F.; NICOLAZZI, E.L.; MACCIOTTA, N.P.P.; Analysis of lactation shapes in extended lactations. Animal, v.6, n.10, p.1572-1582, 2012. https://doi.org/10.1017/S1751731112000766 
THOLON P.; QUEIROZ, S.A. Utilização de diferentes estruturas de variância residual em modelos de regressão aleatória para descrição da curva de crescimento de perdizes (Rhynchotus rufescens) criadas em cativeiro. Caatinga, v.21, n.2, p.37-47, 2008.

WEGMAN, E.J.; WRIGHT, I.W. Splines in statistics. Journal of the American Statistical Association, v.78, n.382, p.351-365, 1983. https://doi.org/10.2307/2288640 